\title{
Análise conjunta de fatores explicativos para o comportamento assimétrico dos custos
}

Joint analysis of explanatory factors for cost stickiness

Análisis conjunto de los factores explicativos para los costos pegajosos

\section{Luiza Santangelo Reis}

Doutora em Contabilidade pela Universidade Federal de Santa Catarina (UFSC)

Professora do Departamento de Ciências Contábeis da UFSC

Endereço: Campus Universitário Reitor João David Ferreira Lima -Trindade

CEP: 88040-900 - Florianópolis/SC - Brasil

E-mail: luizasantangeloreis@gmail.com

Telefone: (48) 3721-6671

\section{Altair Borgert}

Doutor em Engenharia de Produção pela Universidade Federal de Santa Catarina (UFSC)

Professor do Departamento de Ciências Contábeis da UFSC

Endereço: Campus Universitário Reitor João David Ferreira Lima -Trindade

CEP: 88040-900 - Florianópolis/SC - Brasil

E-mail: altair@borgert.com.br

Telefone: (48) 3721-6671

Artigo recebido em 11/05/2018. Revisado por pares em 01/02/2020. Reformulado em 28/03/2020. Recomendado para publicação em 30/03/2020 por Carlos Eduardo Facin Lavarda (Editor-Chefe). Publicado em 30/03/2020. 


\section{Resumo}

O objetivo do presente estudo consiste em determinar a interação conjunta de fatores explicativos relacionados ao decisor e ao ambiente organizacional na assimetria dos custos. Por meio de análise fatorial confirmatória e modelo empírico de regressão os resultados sugerem que os dois clusters afetam positivamente, e em magnitudes semelhantes, a assimetria das despesas gerais, o que comprova a existência de efeitos dos fatores explicativos que se sobrepõem na explicação da assimetria dos custos e corroboram a visão contemporânea sobre gestão de custos causados pela decisão deliberada dos gestores. Ainda, por meio de análise exploratória, revelou-se 6 constructos que resumem as interações dos fatores explicativos da assimetria dos custos. Por fim, conclui-se que esta pesquisa contribui para a compreensão do comportamento dos custos baseada numa visão mais ampla, e não isolada em relação aos fatores explicativos, ao fornecer subsídios para novas teorias e modelos práticos de previsão de lucros aos analistas.

Palavras-chave: Assimetria de custos; Fatores explicativos; Análise conjunta

\section{Abstract}

The study objective is to determine the explanatory factors' joint interaction related to the decision-maker and the organizational environment in the asymmetry of costs. Through confirmatory factor analysis and empirical regression model, the results suggest that the two clusters positively affect, in similar magnitudes, the asymmetry of general and administrative expenses, which proves the existence of the explanatory factors effects that overlap in the sticky behavior explanation and corroborate the contemporary view on cost management caused by the managers' deliberate decision. Still, through exploratory analysis, 6 constructs were revealed that summarize the interactions of the cost asymmetry explanatory factors. Finally, it is concluded that this research contributes to the understanding of cost behavior based on a broader view, and not isolated in relation to explanatory factors, by providing subsidies for new theories and practical models of profit forecasting to analysts.

Keywords: Cost stickiness; Explanatory factors; Joint analysis

\section{Resumen}

El objetivo del presente estudio es determinar la interacción conjunta de factores explicativos relacionados con el tomador de decisiones y el entorno organizacional en los costos pegajosos. A través del análisis factorial confirmatorio y el modelo de regresión empírica, los resultados sugieren que los dos grupos afectan positivamente, en magnitudes similares, la asimetría de los gastos generales, lo que demuestra la existencia de efectos de los factores explicativos que se superponen en la explicación de la asimetría y corroborar la visión contemporánea sobre la gestión de costos causada por la decisión deliberada de los gerentes. Aun así, a través del análisis exploratorio, se revelaron 6 construcciones que resumen las interacciones de los factores explicativos de los costos pegajosos. Finalmente, se concluye que esta investigación contribuye a la comprensión del comportamiento de los costos basado en una visión más amplia, y no aislada en relación con factores explicativos, al proporcionar subsidios para nuevas teorías y modelos prácticos de pronóstico de ganancias a los analistas.

Palabras clave: Costos pegajosos; Factores explicativos; Análisis conjunto

\section{Introdução}

As pesquisas desenvolvidas na área de comportamento dos custos até o início das discussões sobre Sticky Costs, em meados da década de 1990, pressupõem que os custos são fixos ou variáveis, cuja parcela variável se altera simetricamente em relação ao volume e a fixa permanece inalterada para a capacidade instalada (BANKER; BYZALOV, 2014). Em outras palavras, geralmente os autores da época consideravam que as variações dos custos eram 
direcionadas pela amplitude da mudança no nível de atividade, e não pelo seu sentido - aumento ou diminuição.

Contudo, Anderson, Banker e Janakiraman (2003) propuseram uma nova forma de representação do comportamento dos custos que contesta a abordagem tradicional, o qual intitularam de "sticky". Os autores atribuíram aos custos a característica de assimétrico e pegajoso (na tradução literal) para expressar a tendência de aumentarem mais do que reduzirem em comparação a uma mesma variação de receita. No decorrer dos anos seguintes, as pesquisas em comportamento dos custos ganharam espaço na literatura. Prova disso é o paper de Krishnan (2015), editora da revista Journal of Management Accounting Research, que na edição especial comemorativa de 25 anos indicou o tema comportamento dos custos, em especial a assimetria dos custos, a demanda incerta e a estrutura de custos, como o futuro das pesquisas na área gerencial.

Em decorrência da evolução das pesquisas sobre o tema, Malik (2012) desenvolveu um estudo de revisão e síntese literária no qual classifica os trabalhos sobre "cost stickiness" em três níveis sequenciais: (i) evidenciação, (ii) determinação e (iii) consequências. Particularmente, em relação ao segundo nível, Malik (2012) identificou oportunidades para a realização de pesquisas no intuito de identificar novos fatores para explicar a assimetria dos custos, os quais se encontram em desenvolvimento na literatura internacional e nacional.

Nessa direção, pode-se identificar um conjunto de fatores explicativos mais frequentemente explorados sobre o tema, quais sejam: ambiente macroeconômico (BANKER; CIFTCI; MASHRUWALA, 2008; BALAKRISHNAN; LABRO; SODERSTROM, 2014); atraso nos ajustes dos custos (ANDERSON; BANKER; JANAKIRAMAN, 2003; CALLEJA; STELIAROS; THOMAS, 2006; BALAKRISHNAN; LABRO; SODERSTROM, 2014; BANKER; BYZALOV, 2014; BANKER et al., 2014); capacidade operacional ociosa (BALAKRISHNAN; PETERSEN; SODERSTROM, 2004; CALLEJA; STELIAROS; THOMAS, 2006; BALAKRISHNAN; GRUCA, 2008; YASUKATA; KAJIWARA, 2011; CANNON, 2014; SUBRAMANIAM; WEIDENMIER, 2016; AZEEZ; DONGPING; DONATA, 2017; AZEEZ; DONGPING; MAHMOOD, 2018); custos de ajustamento (CHEN; LU; SOUGIANNIS, 2012; DALLAVIA; PEREGO, 2013; BANKER; BYZALOV, 2014; BANKER et al., 2014; LIU; LIU; REID, 2019; CHEN; KAMA; LEHAVY, 2018); decisão deliberada dos gestores (YASUKATA; KAJIWARA, 2011; MALIK, 2012; BLUE et al., 2013; BANKER; BYZALOV, 2014; BANKER et al., 2014); estrutura de custos (BALAKRISHNAN; PETERSEN; SODERSTROM, 2004; CALLEJA; STELIAROS; THOMAS, 2006; BALAKRISHNAN; LABRO; SODERSTROM, 2014); fluxo de caixa livre (ABU-SERDANEH, 2014); intensidade de ativos e passivos (ANDERSON; BANKER; JANAKIRAMAN, 2003; CALLEJA; STELIAROS; THOMAS, 2006; MALIK, 2012; NASSIRZADEH; SAEI; SALEHI, 2013; ABU-SERDANEH, 2014; BALAKRISHNAN; LABRO; SODERSTROM, 2014; CANNON, 2014); magnitude das variações de receitas (CALLEJA; STELIAROS; THOMAS, 2006; MALIK, 2012; SUBRAMANIAM; WEIDENMIER, 2016); otimismo e pessimismo dos gestores (BANKER; CIFTCI; MASHRUWALA, 2008; KAMA; WEISS, 2010; YASUKATA; KAJIWARA, 2011); problemas de agência (CALLEJA; STELIAROS; THOMAS, 2006; CHEN; LU; SOUGIANNIS, 2012; MALIK, 2012; LIANG, 2013; GUENTHER; RIEHL; ROBLER, 2014; NAMITHA; SHIJIN, 2016; LIU; LIU; REID, 2019); regulamentação do mercado (HOLZHACKER; KRISHNAN; MAHLENDORF, 2014); e, tamanho da empresa (BOSCH; BLANDÓN, 2011; NASSIRZADEH; SAEI; SALEHI, 2013).

Vale destacar que tais estudos ocorreram em diferentes momentos (linha temporal) e contextos (ambientes/países), conforme já sintetizado por Richartz e Borgert (2015) e aqui apresentados de forma adaptada. Além disso, devido ao desenvolvimento das pesquisas em diferentes contextos, muitos desses fatores foram testados individualmente e sem vínculo com os demais. Logo, a influência que um fator exerce sobre os demais ainda não foram consideradas em termos literários. 
Porém, com base nos estudos mencionados pode-se inferir, por exemplo, que o fator decisão deliberada dos gestores - que busca compreender o impacto que as decisões otimistas e pessimistas têm sobre a assimetria dos custos - é, em parte, decorrente de um segundo fator, qual seja o ambiente macroeconômico. Nesse contexto, Banker, Fang e Mehta (2013) comprovaram que, embora os custos se mantenham sticky em períodos típicos, durante a crise dos anos 20082009 houve uma reversão significativa para um comportamento anti-sticky, resultado traduzido pelo incomum pessimismo do cenário econômico. Na mesma direção, Banker, Ciftci e Mashruwala (2008) utilizaram o crescimento econômico como proxy para o fator decisão deliberada dos gestores, o que evidencia a interação dos fatores.

Ou seja, os fatores podem interagir entre si, no sentido de representarem conceitos maiores que permeiam grupos de fatores (clusters). Dessa forma, com base na literatura sobre o tema, podese mapear dois grandes clusters no qual um representa o aspecto pessoal do decisor (sujeito), denominado de decisão deliberada dos gestores, e o outro representa a estrutura empresarial (objeto), tratado como atraso nos ajustes de custos.

De modo geral, observa-se que a influência conjunta que os fatores de cada cluster impõem na assimetria dos custos não é considerada nos estudos - devido à avaliação isolada dos mesmos - o que pode afetar a assimetria na medida em que tais fatores explicam "intrinsecamente" o comportamento assumido pelos custos. Em função disso, evidencia-se a necessidade de identificação das influências que tais fatores exercem um sobre os outros e, com base nessas influências, procura-se explicações mais robustas e concisas para a assimetria dos custos. Assim, diante do exposto, tem-se o seguinte problema de pesquisa: Qual a interação dos fatores que, conjuntamente, explica o comportamento assimétrico dos custos? Neste sentido, o objetivo do presente estudo consiste em determinar a interação conjunta de fatores explicativos relacionados ao decisor e ao ambiente organizacional na assimetria dos custos.

A justificativa para o desenvolvimento desta pesquisa está na contribuição para a evolução da teoria de assimetria dos custos que, segundo Malik (2012), ainda necessita de evolução, apesar de sua relevância para a tomada de decisão no que diz respeito à eficiência dos custos. Ainda, segundo a autora, o conceito de cost stickiness não está suficientemente consolidado, talvez pelo fato de existir pouca discussão teórica sobre suas causas e consequências. No contexto prático, a pesquisa se justifica pela possibilidade de previsão dos custos com base em informações disponíveis aos shareholders, uma vez que os modelos de previsão de lucro usados pelos analistas que incorporam o comportamento assimétrico dos custos apresentam menos erros e, desta forma, maior poder preditivo (BANKER; CHEN, 2006; ANDERSON et al., 2007; KIM; PRATHERKINSEY, 2010; BANKER; BYZALOV; FANG; LIANG, 2018).

Por fim, este estudo delimita-se ao contexto brasileiro, especificamente às empresas listadas na Bolsa de Mercadorias e Futuros (BM\&FBovespa). Apesar de ser um fenômeno que pode ocorrer em diversas economias, a maioria dos estudos sobre comportamento assimétrico dos custos se concentra nos Estados Unidos (EUA), país que originou as primeiras pesquisas na área (BANKER; BYZALOV, 2014).

\section{Fundamentação Teórica}

Com base na literatura acerca do tema, em linhas gerais, pode-se estabelecer dois grandes agrupamentos de fatores explicativos (clusters) para o comportamento assimétrico dos custos que representam as dimensões do gestor e da empresa. De acordo com Yasukata e Kajiwara (2011), a assimetria dos custos pode ser explicada por duas perspectivas: a primeira consiste na decisão deliberada dos gestores que compreende, de forma geral, as expectativas diante de demandas futuras; e a segunda denominada atraso nos ajustes de custos, que diz respeito à incapacidade de se ajustar os custos rapidamente para acompanhar as quedas nas receitas. Entretanto, os referidos autores descrevem que essas duas perspectivas não são excludentes entre si, à medida que uma não contesta a outra. 
Nessa direção, Abdulhamied e Abulezz (2012) apoiaram suas análises nas duas dimensões - a primeira decorrente da incerteza na demanda (perspectiva dos gestores) e a segunda da estrutura de custos da organização (perspectiva da empresa). Seus resultados sustentaram as hipóteses de que tanto a estrutura de custos quanto a incerteza na demanda afetam a assimetria dos custos, semelhantemente ao estudo de Yasukata e Kajiwara (2011). Também, a pesquisa de Chen, Kama e Lahavy (2018) se fundamentou nessas perspectivas na medida em que examinaram o efeito das expectativas dos gestores (perspectiva dos gestores) nos cost stickiness em contextos de diferentes níveis de custos de ajustamento dos recursos (perspectiva da empresa).

Como se pode observar, já existe indicação na literatura de uma pré-formatação de duas grandes dimensões, as quais podem ser exploradas com base nos estudos precedentes que propõem interações entre os fatores explicativos para um melhor entendimento sobre o comportamento assimétrico dos custos.

\subsection{Decisão deliberada dos gestores}

Em relação a literatura levantada, um dos fatores explicativos mais citados e discutidos pelos autores é a decisão deliberada dos gestores (YASUKATA; KAJIWARA, 2011; MALIK, 2012; BLUE et al. 2013; BANKER; BYZALOV, 2014; BANKER et al., 2014; BANKER; BYZALOV; FANG, 2018). Este fator explicativo, nesta pesquisa interpretado como uma dimensão latente, compreende as intenções dos gestores mediante incentivos ou cobranças, bem como os aspectos relacionados à expectativa otimista e pessimista dos gestores diante do ambiente macroeconômico.

Existem diversos autores que se utilizam de diferentes justificativas para sustentar o porquê este fator afeta a assimetria dos custos, dentre os quais Yasukata e Kajiwara (2011), que encontraram resultados robustos ao demonstrarem a influência da decisão deliberada dos gestores proveniente do otimismo e pessimismo de acordo com as expectativas de vendas futuras. Para os autores, ao se depararem com situações que não condizem com suas expectativas de vendas, os gestores tomam decisões com base em seu otimismo e pessimismo por acreditarem que tal situação é temporária. Assim, os custos tornam-se mais "stickier" quando os gestores prospectam aumentos de receita em períodos futuros, uma vez que tendem a reter recursos excessivos a curto prazo mesmo diante de atuais quedas na receita (YASUKATA; KAJIWARA, 2011).

Nessa mesma direção, Banker, Ciftci e Mashruwala (2008) identificaram como o otimismo e pessimismo dos gestores afeta a assimetria dos custos, ao utilizar como proxy a sequência de aumentos ou quedas na receita, o crescimento macroeconômico e as ordens de encomendas programadas. Os autores concluíram que, para o planejamento da capacidade produtiva, os gestores observam as tendências macroeconômicas e em períodos de crescimento são mais otimistas em relação às vendas futuras, sobretudo quando o período corrente corresponder à tendência esperada, pois tomam suas decisões de acordo com tais perspectivas.

A observância das tendências macroeconômicas como fator motivador à tendência otimista ou pessimista dos gestores, também, foi objeto de estudo de Banker, Fang e Mehta (2013), em que investigaram se existiu comportamento assimétrico distinto, em relação à crise econômica de 2008, quando ocorreu um período de pessimismo extremo entre os gestores. Como resultado do estudo, os autores mencionam que a crise econômica fez com que muitas empresas sofressem quedas nas vendas, o que causou reavaliações nas expectativas sobre o poder de compra dos consumidores e o crescimento futuro, e levou a um pessimismo quanto às perspectivas de vendas dos períodos seguintes. A magnitude e a velocidade com que a crise ocorreu impactaram o PIB e, por isso, Banker, Fang e Mehta (2013) o utilizaram como proxy para a macroeconomia. Contudo, os autores argumentam que a crise econômica não afetou todas as empresas da mesma forma, mas que aquelas que experimentam um declínio de vendas apresentaram impacto diferente na assimetria. Os resultados sustentam as hipóteses de pesquisa propostas e evidenciam significante comportamento sticky até 2008 e uma reversão significante para anti-sticky durante o período da crise econômica. 
Kama e Weiss (2013) acrescentam outro fator que se relaciona com otimismo e pessimismo dos gestores, qual seja o problema de agência. Os autores identificaram o impacto dos incentivos nos ajustes e estrutura dos custos, e afirmam que os gestores fazem ajustes para otimizar as metas pelas quais recebem incentivos com base em suas crenças na demanda futura decorrente. Assim, caso estes gestores recebam incentivos pela maximização do lucro, mesmo que a receita reduza, cortarão custos a uma taxa mais rápida do que se não recebessem tal incentivo, o que atenua a assimetria dos custos (KAMA; WEISS, 2013).

Ainda, sobre o fator problema de agência, Chen, Lu e Sougiannis (2012) estudaram a sua associação com a assimetria dos custos. Uma das metodologias utilizadas no estudo para mensurar o problema de agência é o fluxo de caixa livre. Isto porque, segundo os autores, diante de aumentos na demanda, a disponibilidade de fluxo de caixa alto permite aos gestores investirem excedentes em despesas e, quando as receitas caem, atrasarem cortes de custos para maximizar os incentivos. Dessa forma, os resultados evidenciaram forte associação entre o fluxo de caixa livre e assimetria dos custos.

Por fim, sobre o enfoque de construção de impérios, e não mais sobre incentivos de lucros, Abu-Serdaneh (2014) testou a hipótese de que o fluxo de caixa livre é positivamente associado com o comportamento assimétrico dos custos e citou Jensen (1986) para argumentar que gestores que têm a sua disposição fluxo de caixa livre são mais propensos a investir em operações ou projetos com valor presente líquido negativo do que a pagar seus acionistas para aumentar suas gratificações. Ainda, Abu-Serdaneh (2014) argumenta, com base em outros autores, que os gestores tendem a aumentar as empresas além do seu tamanho ótimo para aumentar a utilidade própria em prol do status, poder e prestígio (problema de agência).

Em função dos estudos mencionados, é possível perceber que há interação entre os fatores: decisão deliberada dos gestores, ambiente macroeconômico, pessimismo e otimismo dos gestores, problema de agência e fluxo de caixa livre. Tal interação é, ainda, mais evidente nos casos em que um fator funciona como proxy do outro, conforme exposto anteriormente. Consequentemente, sugere-se que essas relações apresentam uma dimensão latente nos fatores propostos para explicar o comportamento assimétrico dos custos nas empresas. Em outras palavras, tais fatores podem ser responsáveis por explicar conjuntamente uma mesma parcela da assimetria dos custos.

\subsection{Atraso nos ajustes de custos}

Neste segundo agrupamento, alguns estudos propõem interação de fatores relacionados ao atraso nos ajustes de custos, ou seja, aqueles fatores relacionados as características organizacionais e sua estrutura de custos, que impedem que os custos se ajustem rapidamente diante de quedas na receita. Neste sentido, Banker e Byzalov (2014) descrevem a pré-disposição dos gestores em manter recursos não utilizados (capacidade ociosa) em períodos de queda da receita para evitar os custos de ajustamento. Nos períodos de aumento de receita, segundo os autores, os custos de ajustamento também diminuem com base na disposição dos gestores em expandir os recursos, porém com menor intensidade, uma vez que, sem o aumento de capacidade, não é possível acomodar o aumento das vendas. No seu estudo, os autores utilizaram como uma das proxies para o custo de ajustamento a intensidade de funcionários, pois exigem custos como os admissionais, de formação para novas contratações, com encargos sobre demissões entre outros. Os resultados evidenciaram que a assimetria dos custos em organizações de diversos países varia conforme os seus custos de ajustamento.

Balakrishnan, Petersen e Soderstrom (2004) testaram se a capacidade operacional e a magnitude das variações dos níveis de atividade afetam a assimetria dos custos, e sugerem que o comportamento dos custos é mais susceptível às grandes mudanças nos níveis de atividades do que a pequenas. Descrevem, ainda, que a capacidade atual utilizada da entidade pode afetar a decisão dos gestores frente a tais mudanças. Assim, segundo os autores, se a organização operar com capacidade ociosa, os gestores podem ser mais propensos a utilizar essa folga operacional para absorver o aumento de demanda. Logo, a resposta ao aumento de demanda é menor que a 
redução. Resumidamente, com 1.898 observações mensais em 49 clínicas de terapia, os autores encontraram uma interação significante entre a capacidade utilizada e a assimetria para diferentes níveis da magnitude de variação da receita.

Semelhantemente, Calleja, Steliaros e Thomas (2006) sustentam que a assimetria dos custos reduz com o aumento da magnitude das variações de receita. Argumentam, ainda, que diante de aumentos de baixas proporções nas receitas - até 10\% - as empresas conseguem acomodar essas variações com a capacidade e recursos que já possuem e, para as reduções de mesmas proporções, o custo de manter a capacidade ociosa é menor do que possíveis custos de renegociação (custos de ajustamento). Por outro lado, em grandes proporções de variação de receita - entre $10 \%$ e $50 \%$ - o custo de manter a capacidade ociosa e os recursos excedentes supera os custos de renegociá-los posteriormente. Dessa forma, torna-se vantajoso desfazer de tais custos, o que gera significante diminuição na assimetria.

Sob a ótica interna da entidade, com base em 189 hospitais de Ontário (Canadá), Balakrishnan e Gruca (2008), também, inferem que o aumento/redução da capacidade operacional é influenciado pela intensidade e especialidade dos ativos. Os autores comparam os departamentos essenciais envolvidos no atendimento direto aos pacientes e os de apoio, tais como lavanderia e dietético. A hipótese sustentada pelos autores é decorrente do argumento que os departamentos de atendimento direto aos pacientes exigem ativos específicos, como pessoal especializado e equipamentos sofisticados, o que dificulta o ajuste da capacidade de maneira rápida no curto prazo. Os resultados são consistentes e evidenciam que os custos dos departamentos de serviços diretos são mais assimétricos comparativamente aos de apoio e auxiliares.

Outro fator, levantado por Nassirzadeh, Saei e Salehi (2013), é o tamanho da empresa, que se relaciona com a estrutura de custos, no que se refere à intensidade de ativos, por meio de um estudo sobre a assimetria dos custos das empresas industriais listadas na bolsa de valores do Teerã. Numa de suas hipóteses sobre os fatores que causam tal comportamento, testaram se o tamanho dessas organizações afeta positivamente a assimetria dos custos. Como proxy para sistematizar o tamanho das organizações utilizaram a intensidade de ativos em relação às vendas totais, cuja escolha metodológica, em termos de definição de variáveis, caracteriza o estreitamento na relação desses dois fatores. Em outras palavras, a hipótese formulada pelos autores sustentava que quanto maior a proporção de ativos em relação ao total de vendas (tamanho da empresa), maior seria a assimetria dos custos. Contudo, os dados da população analisada pelos autores rejeitaram a hipótese tanto para os custos dos produtos vendidos quanto para as despesas gerais, administrativas e de vendas.

Estudos mais recentes como os de Azeez, DongPing e Donata (2017) e Azeez, DongPing e Mahmood (2018), também, evidenciaram que o impacto de fatores de capacidade, como problemas complexos de estimativas de capacidade, custos de ajustamento e nível de utilização da capacidade do período corrente e anteriores afetam o comportamento assimétrico dos custos, inclusive os custos com mão de obra. Percebe-se, diante do exposto, que a literatura propõe certas relações entre os fatores ligados ao atraso nos ajustes de custos quais sejam: a magnitude das variações de receita, a capacidade operacional, a intensidade de ativos e passivos, o tamanho da empresa e os custos de ajustamento.

\section{$3 \quad$ Procedimentos Metodológicos}

O ambiente de estudo se caracteriza, na economia brasileira, pelas empresas negociadas na BM\&FBovespa durante o período de 2010 a 2014, as quais foram selecionadas por apresentarem as informações necessárias para a realização da presente pesquisa. Excluiu-se dessa população as empresas do setor financeiro, uma vez que possuem planos de contas específicos e particularidades referentes a regulamentações, o que inviabiliza a sua comparabilidade com os demais setores. Além dessas, com o intuito de viabilizar as análises estatísticas, excluiu-se também os anos (períodos) das empresas que não apresentaram as informações para todos os fatores explicativos. Diante disso, por existirem fatores explicativos que exigem informações de caixa e equivalente de 
caixa, as quais constam no sistema Economática somente a partir de 2010, este foi definido como ano de corte inicial. Já, quanto a exclusão de outliers, identificou-se que a maioria dos estudos sobre o tema utiliza como critério de exclusão valores com variação de mais de $50 \%$ nas receitas no período de um ano, o que também foi observado. Ao final desses procedimentos, chegou-se a 163 empresas com 617 observações, as quais fazem parte das análises do presente estudo.

Com base nos dados da Economática, e de acordo com os parâmetros anteriormente citados, levantou-se para as empresas selecionadas os dados financeiros necessários, conforme proxies especificadas mais adiante. As informações referentes aos gastos com pessoal foram coletadas por meio da Demonstração do Valor Adicionado (DVA), disponível no site da BM\&FBovespa. Já a série histórica do PIB corrente foi obtida no site do IBGE. Todas essas informações foram levantadas nas demonstrações encerradas em 31 de dezembro de cada ano e atualizadas pelo índice IPCA. A análise dos dados é feita em três etapas: na primeira efetua-se uma análise global da assimetria com base nos custos totais (CT) que compreende o custo dos produtos vendidos (CPV), somadas as despesas de vendas (DV) e as despesas administrativas (DA); num segundo momento analisa-se, particularmente, o comportamento referente ao CPV; e por fim, apresenta-se uma análise conjunta das DV com as DA, denominadas de Despesas Gerais (DG).

Uma das possíveis abordagens para o alcance do objetivo de pesquisa proposto, em termos de análise conjunta dos fatores explicativos, é a análise fatorial, cuja abordagem fornece ferramentas para analisar a estrutura das interrelações entre os fatores explicativos do comportamento assimétrico dos custos. Dessa forma, a análise fatorial retorna os scores fatoriais que representam as dimensões latentes que, no caso da presente pesquisa, são os clusters, a partir dos quais pode-se fazer o uso da análise própria de assimetria proposta por Anderson, Banker e Janakiraman (2003) e verificar sua significância em relação ao modelo.

A Figura 1explicita as variáveis inseridas (retângulos) no modelo de análise fatorial confirmatória (fatores explicativos) e os respectivos clusters (dimensão latente - pelas elipses). Ainda, vale ressaltar que na análise fatorial os clusters são o output da análise fatorial (escores fatoriais) por representarem as dimensões latentes dos fatores explicativos que os compõem.

As proxies evidenciadas na Figura 1 foram elencadas com base na literatura proposta da fundamentação teórica. Vale destacar que para a inserção na análise fatorial todas as proxies, com exceção das variáveis dummy, foram transformadas para seu correspondente em log., com o propósito de reduzir a variabilidade dos dados na medida em que há grande diversidade entre as medidas adotadas. Assim, por meio da Análise Fatorial Confirmatória é possível identificar qual dos fatores contribui mais ou menos na formação dos clusters, informação relevante para a gestão das empresas.

Num segundo momento, exportou-se essas variáveis estatísticas dos clusters (output da análise fatorial) e as inseriu-se como variáveis independentes na análise de regressão com dados em painel - modelo consolidado para testar a assimetria dos custos. Consequentemente, com base no modelo proposto por Anderson, Banker e Janakiraman (2003), e com adequações necessárias, definiu-se como variáveis independentes as variações de receita, o cluster de decisão deliberada dos gestores e o cluster de atraso nos ajustes de custos para cada uma das variáveis dependentes de CPV, DG e CT. 
Figura 1- Fatores Explicativos e Proxies correspondente

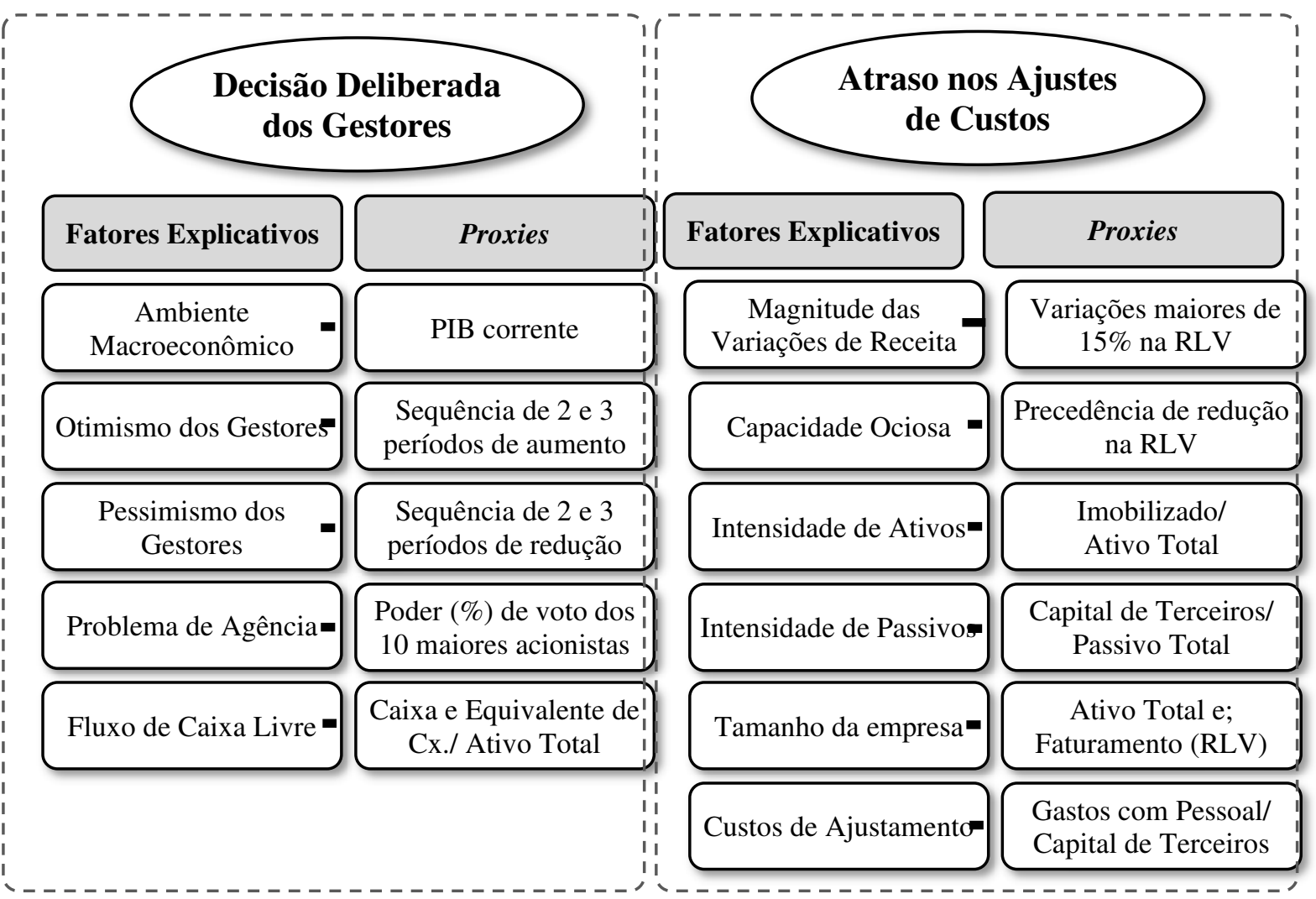

Fonte: Elaborado pelos autores, com base nos dados da pesquisa

\section{Apresentação e Análise dos Dados}

O desenvolvimento do presente estudo acontece em três etapas. A primeira tem por objetivo a construção e avaliação dos clusters sugeridos pela literatura por meio da análise fatorial confirmatória. A segunda determina o impacto destes clusters na assimetria dos custos dos produtos vendidos, despesas gerais e custos totais. Já, a terceira objetiva a verificação e determinação de clusters por meio da estatística, independentemente da literatura, por meio da análise fatorial exploratória.

Destaca-se que ambos os clusters propostos - Decisão Deliberada dos Gestores e Atraso nos Ajustes de Custos - são caracterizados como a consequência dos demais indicadores, o que evidencia uma relação do tipo formativa. Os fatores explicativos que possuem mais de uma variável de medida como é o caso do otimismo e pessimismo dos gestores, bem como o tamanho das organizações, são tratados inicialmente como uma dimensão latente do tipo reflexivo. Num segundo momento, estas dimensões reflexivas são substituídas pelos scores latentes provenientes de uma primeira "rodada" do software. Este procedimento de duas fases é sugerido no trabalho de Lowry e Gaskin (2014) em modelos com constructos de segunda ordem (uma dimensão latente predizendo outra) como ocorre no presente modelo. Assim, diante dos modelos sugeridos extraiuse, os pesos e loadings provenientes da análise fatorial confirmatória na Figura 2, bem como os seus graus de significância.

Com base na Figura 2, percebe-se que dentre as características estruturais que acarretam atraso nos ajustes de custos são os custos de ajustamento, que mais impactam nesta dimensão $(0,938)$. Assim, em empresas em que é reconhecida a presença de muitos custos de ajustamento para se alterar a capacidade instalada, maior é o seu impacto na assimetria comparativamente a outros fatores como o tamanho das organizações $(-0,466)$ ou a intensidade de ativos $(-0,140)$. Estes resultados corroboram os argumentos discutidos nas pesquisas de Banker e Byzalov (2014) de que 
a presença de custos de ajustamento de recursos nas empresas inibe os gestores de ajustarem a capacidade, bem como ratificam os achados de Calleja, Steliaros e Thomas (2006), Azeez, DongPing e Donata (2017) e Azeez, DongPing e Mahmood (2018) discutidos na fundamentação teórica.

Figura 2- Indicadores dos clusters

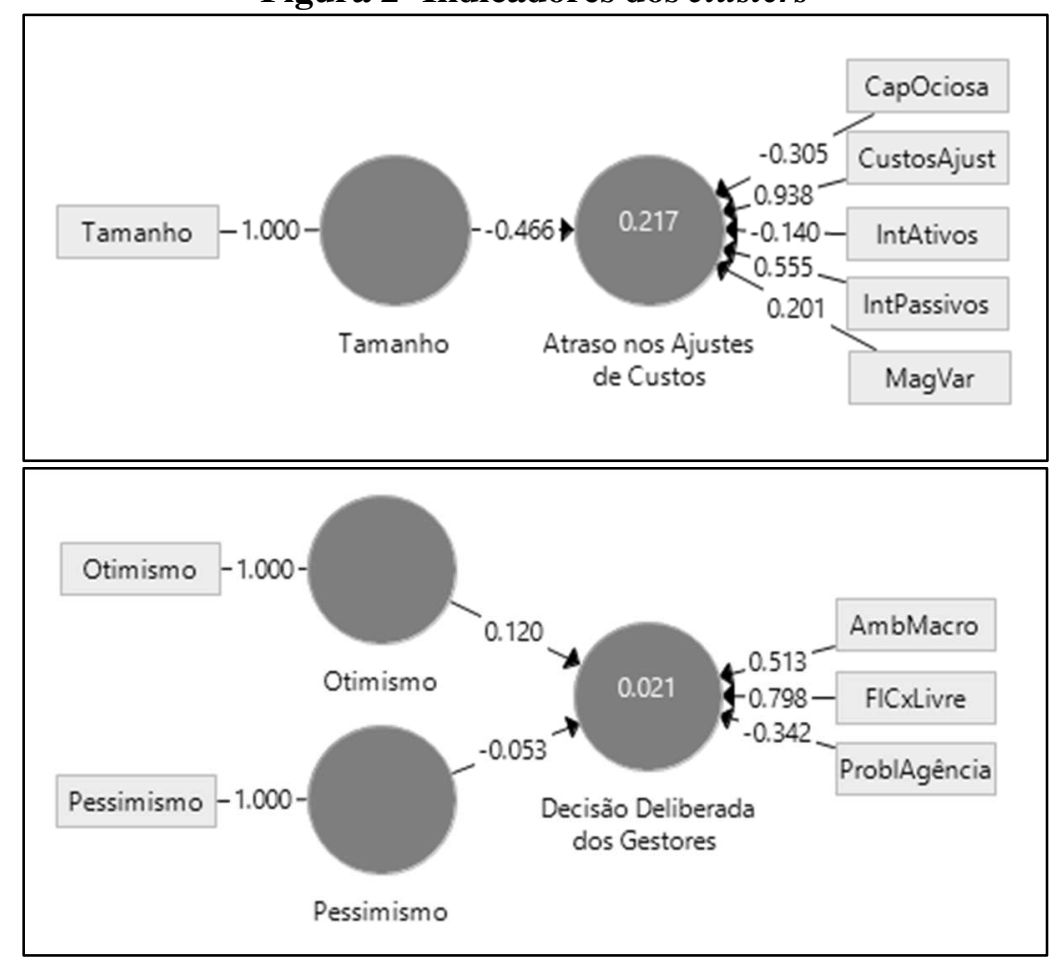

Fonte: Elaborado pelos autores, com base nos dados da pesquisa

Já, para a dimensão do decisor, o fator explicativo de maior intensidade é o fluxo de caixa livre $(0,798)$. Assim, mesmo que os gestores apresentem tendências otimistas $(0,120)$ ou pessimistas $(-0,053)$, o fato de haver ou não fluxo de caixa livre é ainda mais impactante no comportamento assimétrico dos custos. Os resultados reafirmam os argumentos de Chen, Lu e Sougiannis (2012) e Abu-Serdaneh (2014) de que os gestores que, diante de aumentos de receitas, dispõem de fluxo de caixa livre são propensos a investirem esses valores excedentes em despesas e projetos com valor líquido negativo.

Deu-se continuidade às análises dos dados após o procedimento de bootstrapping, o qual retorna os valores das significâncias dos indicadores até então analisados. Com base nessas informações, inferiu-se que o indicador de fluxo de caixa livre é significativo a um grau de 97,68\% de confiança. Já, o constructo de ambiente macroeconômico alcançou significância a um grau de $94,52 \%$ de confiança, seguido pelo otimismo dos gestores com 83,24\%. Para os indicadores de pessimismo dos gestores e problema de agência esses valores foram de 64,76\% e 63,72\% respectivamente. Para o cluster de atraso nos ajustes de custos observa-se que todos os indicadores, com exceção da intensidade de ativos, possuem significância a um grau de mais de $95 \%$ de confiança se aproximando a $100 \%$. E, a intensidade de ativos possui significância a 90,70\% de confiança, visto que seu valor t é de 1,687. Esses resultados comprovam a existência de efeitos dos fatores explicativos que se sobrepõem na explicação da assimetria dos custos.

Apesar de alguns indicadores apresentarem valores baixos em termos de grau de confiança, possuem características de não serem intercambiáveis devido a este construto ser formativo, ou seja, remover ou reposicionar qualquer um dos indicadores pode trocar o significado do cluster (LOWRY; GASKIN, 2014). Em outras palavras, não é possível mensurar decisão deliberada dos gestores sem ponderar eventuais problemas de agência em seu ambiente organizacional, ou sua postura otimista ou pessimista e assim por diante. Diante disso é necessário manter todos os indicadores no modelo para caracterizar corretamente o cluster decisão deliberada dos gestores. 
Até este ponto, analisou-se a estrutura do modelo de mensuração refletido no mapeamento da literatura existente, bem como os pesos que os fatores explicativos da assimetria possuem sobre os clusters relacionados a aspectos do decisor e da estrutura operacional. Na sequência, utilizouse os scores (valores) latentes dos clusters como variáveis independentes no modelo proposto por Anderson, Banker e Janakiraman (2003) a fim de determinar quais seus impactos no comportamento dos custos. Esta parte da análise objetiva determinar a influência que os clusters de atraso nos ajustes de custos e de decisão deliberada dos gestores possuem sobre a assimetria dos custos.

Uma vez que a existência, bem como a comprovação do comportamento assimétrico dos custos nas indústrias brasileiras, para o período de interesse, já foram verificadas em diversos estudos (MEDEIROS; COSTA; SILVA, 2005; RICHARTZ; BORGERT, 2014; RICHARTZ; BORGERT; LUNKES, 2014; PAMPLONA et al., 2015; KREMER, 2015), na presente análise aceitou-se a existência do comportamento assimétrico como verdadeiro e concentrou-se na análise da influência dos clusters neste comportamento. Com este propósito, buscou-se a determinação desses dois clusters na assimetria, que por sua vez é configurada em três itens: custo dos produtos vendidos (CPV), despesas de vendas e administrativas (DG) e a soma desses dois itens que caracterizam os custos totais (CT).

Após as análises dos pressupostos dos modelos de regressão, passou-se aos resultados dos modelos de assimetria dos três itens de custos. Portanto, inicia-se as análises do comportamento assimétrico para o CPV conforme exposto na Tabela 1. Para estas análises considera-se 90\% o grau de confiança aceitável aos achados da pesquisa, ou seja, probabilidade de t menor ou igual a 0,10 para todos os coeficiente.

\section{Tabela 1 - Assimetria do CPV, DG e CT}

\begin{tabular}{|c|c|c|c|c|c|c|c|c|c|}
\hline & & & & \multicolumn{3}{|c|}{ Robust } & \multicolumn{3}{|c|}{ Semirobust } \\
\hline & $\begin{array}{l}\text { Coef. } \\
\text { CPV }\end{array}$ & $\begin{array}{l}\text { Std Err. } \\
\text { CPV }\end{array}$ & $\begin{array}{l}\mathrm{P}>|\mathrm{t}| \\
\mathrm{CPV}\end{array}$ & $\begin{array}{c}\text { Coef. } \\
\text { DG }\end{array}$ & $\begin{array}{l}\text { Std Err. } \\
\text { DG }\end{array}$ & $\begin{array}{l}\mathrm{P}>|\mathrm{t}| \\
\mathrm{DG}\end{array}$ & $\begin{array}{l}\text { Coef. } \\
\text { CT }\end{array}$ & $\begin{array}{c}\text { Std Err. } \\
\text { CT }\end{array}$ & $\begin{array}{l}\mathrm{P}>|\mathrm{t}| \\
\mathrm{CT}\end{array}$ \\
\hline VarRLV & 0.8240 & 0.0638 & 0.000 & 0.4599 & 0.1009 & 0.000 & 0.8619 & 0.0447 & 0.000 \\
\hline DDRL & -0.1346 & 0.1102 & 0.223 & 0.0455 & 0.1442 & 0.752 & -0.2007 & 0.1054 & 0.058 \\
\hline Decisão Gestores & -0.0805 & 0.0454 & 0.077 & -0.1901 & 0.0653 & 0.004 & -0.1030 & 0.0587 & 0.080 \\
\hline Atraso Custos & 0.0096 & 0.6636 & 0.885 & -0.1950 & 0.8920 & 0.029 & -0.0474 & 0.1088 & 0.663 \\
\hline _cons & -0.0002 & 0.0029 & 0.951 & -0.0009 & 0.0037 & 0.805 & -0.0015 & 0.0029 & 0.594 \\
\hline R-sq overall & 0.5274 & Prob $>F$ & 0.000 & 0.2109 & Prob $>$ F & 0.000 & Pro & $b>F$ & 0.000 \\
\hline \multicolumn{9}{|c|}{ Durbin-Watson statistic (original) } & 17.096 \\
\hline \multicolumn{9}{|c|}{ Durbin-Watson statistic (transformed) } & 18.869 \\
\hline \multicolumn{9}{|l|}{ R-square } & 0.6521 \\
\hline
\end{tabular}

Fonte: Elaborado pelos autores, com base nos dados da pesquisa

A Tabela 1 apresenta os resultados para a análise da influência dos clusters de atraso nos ajustes de custos (estrutura operacional) e decisão deliberada dos gestores (decisor) no comportamento assimétrico dos custos dos produtos vendidos (CPV), das despesas gerais (DG) e dos custos totais (CT). Conforme o modelo de assimetria desenvolvido por Anderson, Banker e Janakiraman (2003), a intensidade da influência dos clusters na assimetria se dá pelo valor do coeficiente e o sentido desta influência é dado pelo sinal. Assim quando o sinal é negativo, maior é a assimetria no sentido sticky e, logo, o cluster está positivamente associado com a assimetria.

Dessa forma, pode-se inferir que apenas o cluster de decisão deliberada dos gestores é significante $(\mathrm{P}<=0,10)$ na influência do comportamento assimétrico do CPV. Esta influência é de 0,0805 positivamente associada à assimetria. Em outras palavras, uma empresa em que o decidor tome decisões do tipo deliberada em benefício próprio, ou conforme suas disposições otimistas 
sobre o futuro o CPV, reduz 8,05p.p. menos em períodos de queda na receita do que para as demais empresas.

Ao mesmo tempo, expandiu-se a mesma análise para as despesas gerais (DG),em que os resultados tanto do cluster decisão deliberada dos gestores quanto do atraso nos ajustes de custos mostraram-se significativos a $90 \%$ de confiança $(\mathrm{P}>|\mathrm{t}|<=0,10)$ e negativos o que evidencia sua associação positiva com a assimetria. Neste caso, quanto mais as empresa têm uma estrutura operacional engessante em que ocorra atraso nos ajustes de custos maior é sua assimetria de custos. Esse aumento na assimetria das despesas gerais é 19,50p.p. comparativamente àquelas em que não ocontece atraso quando há quedas nas receitas. Já, os aumentos nas despesas gerais, para $1 \%$ de aumento nas receita, é de $0,46 \%$ independente da presença de estrutura engessante ou não.

Quanto à influência da decisão deliberada dos gestores na assimetria das despesas gerais, esta é de 0,1901 em mesmo sentido daquela. Assim, para as empresas em que os gestores tomam decisões deliberadamente ocorre retração na queda das despesas gerais de $0,1901 \%$ para reduções de $1 \%$ na receita comparativamente àquelas em que os gestores tomam decisões pautadas em evidências. Dessa forma, ao comparar a influência que a decisão deliberada dos gestores exerce sobre a assimetria com a influência dos atrasos nos ajustes de custos é aproximadamente a mesma para as despesas gerais, com uma diferença de 0,005p.p. a mais para os aspecto estrutural.

Como os CT são a soma dos CPV com as DG, espera-se um comportamento semelhante em termos de sentido e intensidade da influência que os clusters exercem sobre o comportamento assimétrico deste item de custo. Ao observar a Tabela 1, percebe-se sua maior semelhança aos resultados de CPV, uma vez que apenas o cluster de decisão deliberada dos gestores mostrou-se significativo a $90 \%$ de confiança e a intensidade deste de 0,1030 . Neste caso, a assimetria dos CT para as empresas em que ocorre deciões deliberadas por parte dos gestores incrementa este comportamento em 10,30p.p. diante de quedas na receita, comparativamente àquelas em que tal situação não ocorre.

A fim de tornar visível os resultados alcançados até o presente momento, elabora-se a Figura 3 com um resumo dos achados, em que é possível visualizar as influências exercidas na assimetria desde os fatores explicativos até os clusters propostos.

Observa-se, na Figura 3, o grau ordenado de influência que os fatores explicativos da assimetria dos custos exercem sobre a formação dos clusters de atraso nos ajustes de custos e de decisão deliberada dos gestores, bem como o impacto igualitário destes no comportamento assimétrico das despesas gerais. Para a assimetria dos custos totais e custos dos produtos vendidos não foi possível uma comparação da intensidade no impacto dos dois clusters devido a somente o cluster relacionado ao gestor ter se mostrado significativo. Diante desses achados é possível estabelecer que estes dois grandes agrupamentos de fatores possuem impacto na assimetria e, ainda, que este impacto ocorre de forma igualitária. Esses resultados evidenciam uma visão ampla e permitem ao decisor a percepção de que, não só sua postura na tomada de decisão acarreta comportamento assimétrico, mas que o mesmo também ocorre devido às características da estrutura organizacional.

Por fim, numa terceira etapa, objetiva-se o desenvolvimento de uma análise exploratória dos fatores explicativos da assimetria dos custos, de modo que não sejam consideradas as interações propostas pela literatura e sim a determinação de constructos latentes por meio da estatística. Dessa forma, em posse dos constructos determinados pelo modelo de análise fatorial exploratória (EFA) podem-se estabelecer comparações entre estes e os dois clusters mapeados na literatura prévia. Destaca-se, ainda, que para atingir a comparabilidade os fatores explicativos e suas proxies são as mesmas para ambas as análises.

Inicialmente, fez-se a análise dos pressupostos para verificar a adequação dos dados ao uso da estatística proposta. Neste sentido, os dados foram submetidos às estatísticas KMO (KaiserMeyer-Olkin), esfericidade de Bartlett, MSA (Measure of Sampling Adequacy) e análise das comunalidades. Assim, a estatística KMO apresentou-se aceitável $(\mathrm{KMO}=0,555)$ e o teste de 
esfericidade de Bartlett indicou a existência de correlações significativas entre as variáveis originais, o que corrobora a aplicação da análise fatorial (FÁVERO et al., 2009).

Figura 3- Influência dos fatores e clusters na assimetria

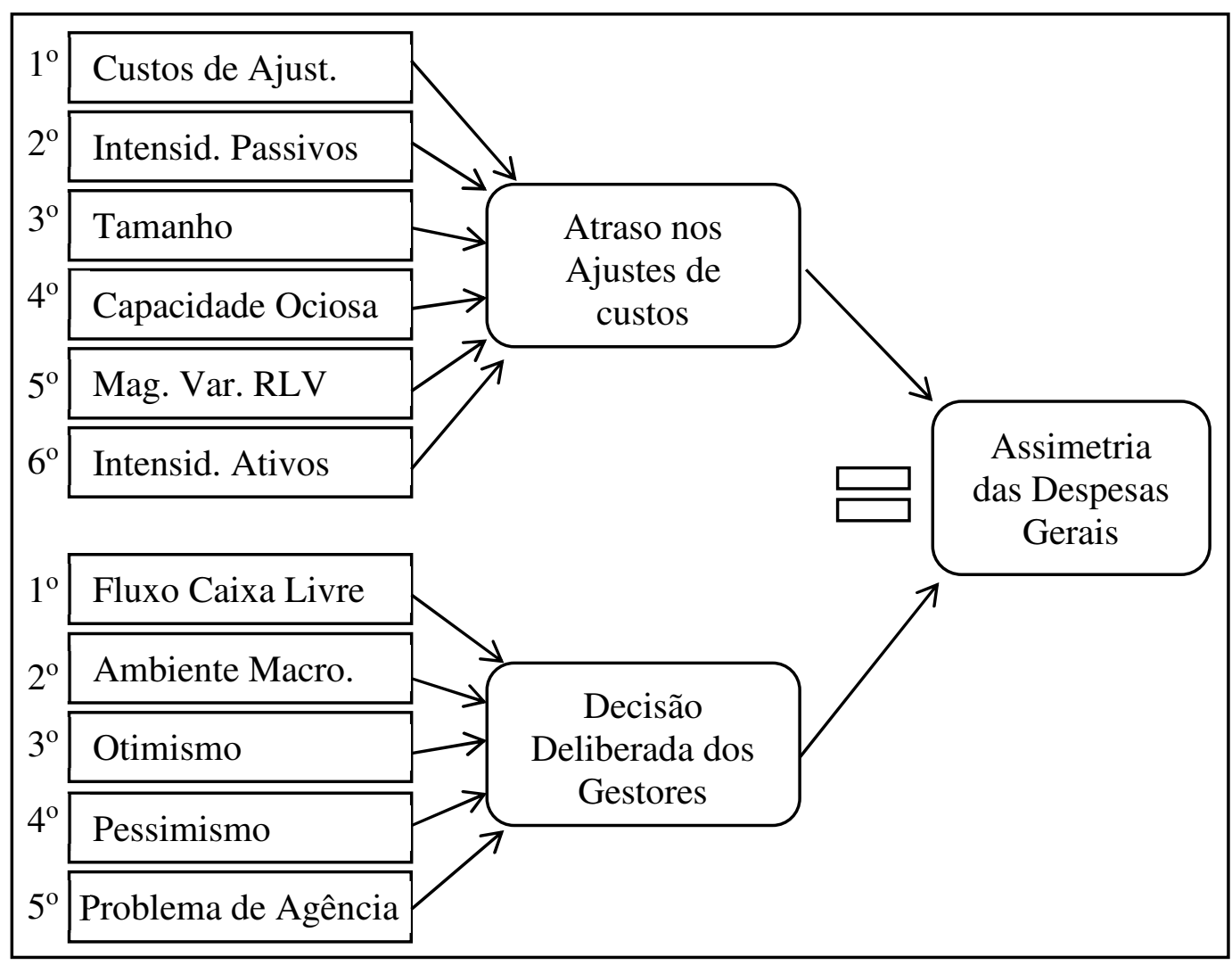

Fonte: Elaborado pelos autores

Os dados também se mostraram adequados nas análises dos parâmetros da Matriz antiimagem e da Medida de adequação da amostra (MAS). Estas análises são reforçadas pelos resultados das comunalidades, que representam a parte da variância compartilhada entre os fatores explicativos da assimetria inseridos na análise (HAIR et al., 2009; FÁVERO et al., 2009). Os resultados para este teste evidenciam que as dimensões latentes extraídas do modelo explicam a maior parte da variância total compartilhada entre os fatores explicativos, com exceção do fluxo de caixa livre $(0,413)$, do ambiente macroeconômico $(0,443)$ e da magnitude das variações da receita $(0,457)$. Contudo, para se obter comparabilidade com os achados anteriores, manteve-se todas as variáveis na análise. Assim, ainda, sobre a análise da variância total explicada apresentase a Tabela 2, que evidencia o número de dimensões extraídas (constructos) e os percentuais de variâncias explicadas por cada uma destas.

Com base na Tabela 2 verifica-se a retenção de 6 dimensões (com autovalores superiores a 1) e o poder de explicação da variância de cada uma delas. Assim, infere-se que a dimensão 1 explica $14,52 \%$ da variância total das 14 variáveis inseridas, após a rotação, e assim por diante. A mesma análise pode ser ampliada para as demais dimensões. Dessa forma, estas 6 dimensões latentes possuem um poder de explicação de $63,76 \%$ do total da variância dos fatores explicativos da assimetria dos custos mensurados nas 14 proxies inseridas nas análises. 
Tabela 2 - Variância total explicada

\begin{tabular}{|c|c|c|c|c|c|c|}
\hline \multirow{2}{*}{ 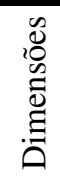 } & \multicolumn{3}{|c|}{ Valores próprios iniciais } & \multicolumn{3}{|c|}{$\begin{array}{c}\text { Somas rotativas de carregamentos ao } \\
\text { quadrado }\end{array}$} \\
\hline & Total & \% de variância & $\%$ cumulativa & Total & \% de variância & $\%$ cumulativa \\
\hline 1 & 2,324 & 16,601 & 16,601 & 2,033 & 14,525 & 14,525 \\
\hline 2 & 1,739 & 12,424 & 29,025 & 1,920 & 13,713 & 28,237 \\
\hline 3 & 1,413 & 10,095 & 39,120 & 1,372 & 9,799 & 38,037 \\
\hline 4 & 1,273 & 9,091 & 48,211 & 1,340 & 9,569 & 47,606 \\
\hline 5 & 1,174 & 8,385 & 56,596 & 1,238 & 8,840 & 56,446 \\
\hline 6 & 1,003 & 7,165 & 63,762 & 1,024 & 7,316 & 63,762 \\
\hline 7 & 948 & 6,774 & 70,535 & & & \\
\hline 8 & ,924 & 6,601 & 77,137 & & & \\
\hline 9 &, 843 & 6,022 & 83,159 & & & \\
\hline 10 & ,748 & 5,344 & 88,504 & & & \\
\hline 11 &, 561 & 4,005 & 92,509 & & & \\
\hline 12 &, 517 & 3,692 & 96,201 & & & \\
\hline 13 & ,323 & 2,309 & 98,511 & & & \\
\hline 14 & 209 & 1,489 & 100,000 & & & \\
\hline
\end{tabular}

Fonte: Elaborado pelos autores, com base nos dados da pesquisa

De maneira complementar, apresenta-se na Tabela 3 a matriz dos componentes, após a rotação, que permitem a visualização à qual dimensão cada um dos fatores explicativos do comportamento assimétrico dos custos está mais associado.

Tabela 3 - Matriz de componente rotativa

\begin{tabular}{l|r|r|r|r|r|r}
\hline & \multicolumn{7}{|c}{ Componentes } \\
\cline { 2 - 7 } & 1 & \multicolumn{1}{c}{2} & \multicolumn{1}{c}{3} & \multicolumn{1}{c}{4} & \multicolumn{1}{c}{5} & \multicolumn{1}{c}{6} \\
\hline FlCxLivre &, 065 &,- 073 &, 084 & $\mathbf{, 5 8 9}$ &,- 061 &,- 214 \\
IntAtivos &, 065 &, 038 & $\mathbf{, 7 0 9}$ &,- 027 &, 466 &, 206 \\
IntPassivos &, 021 &, 197 &,- 088 &,- 039 & $\mathbf{, 6 7 3}$ &,- 198 \\
CustosAjust &, 024 &,- 059 & $\mathbf{, 7 8 0}$ &, 195 &,- 260 &,- 041 \\
MagVar &,- 103 &,- 084 &,- 031 & $\mathbf{- , 6 3 2}$ &, 073 &,- 183 \\
Pess2p &,- 172 & $\mathbf{, 8 7 6}$ &, 021 &,- 060 &, 055 &,- 032 \\
Pess3p &,- 102 & $\mathbf{, 8 2 8}$ &,- 027 &,- 026 &, 057 &, 051 \\
CapOciosa & $\mathbf{- , 6 5 4}$ &,- 487 &,- 087 &,- 134 &, 097 &, 065 \\
Otim2p & $\mathbf{, 8 9 2}$ &,- 242 &, 000 &, 043 &,- 016 &,- 009 \\
Otim3p & $\mathbf{, 8 5 3}$ &,- 194 &, 000 &, 035 &, 061 &,- 005 \\
ProblAg &,- 057 &, 071 &, 044 &, 065 &,- 217 & $\mathbf{, 8 0 5}$ \\
AmbMacro &, 035 &,- 218 &,- 079 &,- 260 &, 386 &, 414 \\
TamRL &,- 128 &,- 129 &,- 027 & $\mathbf{5 0 2}$ &, 486 &,- 077 \\
TamAT &, 044 &,- 094 &,$- \mathbf{4 7 7}$ &, 453 &, 199 &, 167 \\
\hline Método de extração: Análise do Componente principal. \\
Método de rotação: Varimax com normalização de Kaiser. \\
Rotação convergida em 12 iteraç̃̃es. \\
\hline Fonte: Elaborado pelos autores, com base nos dados da pesquisa
\end{tabular}


$\mathrm{Na}$ Tabela 3, os componentes numerados representam as dimensões e os valores são as cargas (loadings). Segundo Fávero et al. (2009), os loadings correlacionam as variáveis (fatores explicativos do comportamento assimétrico) com as dimensões aqui já extremadas pelo método de rotação ortogonal utilizado. Ainda, sobre a explanação destes autores, a rotação ortogonal, além de gerar dimensões não correlacionadas entre si, extrema os loadings de modo que cada fator se vincule majoritariamente a uma dimensão, conforme os realces na Tabela 3.

Dessa forma, numa análise da Tabela 3, pode-se inferir que a dimensão 1 é composta predominantemente pelo fator otimismo dos gestores, para 2 e 3 períodos, e capacidade ociosa no sentido em que para a sua ocorrência os gestores devem estar otimistas em relação ao futuro por manterem recursos ociosos em vez de se desfazerem de tais recursos. Já, a dimensão 2 compreende o pessimismo dos gestores para 2 e 3 períodos sequenciais de queda na receita. A terceira dimensão é formada majoritariamente pela intensidade de ativos, custos de ajustamento e tamanho das organizações com relação aos ativos totais. Tal associação pode ser descrita de forma que, quanto maior for o tamanho da empresa em termos de ativo, o grau em que ocorre a imobilização e consequentemente a intensidade de ativos também cresce, enquanto que por apresentar estrutura complexa o número e gastos com pessoal tende a subir com aumento nos custos de ajustamento. A dimensão 4 inclui, predominantemente, os fatores de fluxo de caixa livre, magnitude das variações da receita e tamanho das organizações com base no seu faturamento. Para a dimensão 4 não há, aparentemente, descrição na literatura que sustente tal associação. Por fim, as duas últimas dimensões são definidas, basicamente, por intensidade de passivos e problema de agência, respectivamente.

A análise fatorial permitiu que fossem extraídas dimensões que representam o agrupamento, e resumem os fatores explicativos (14 proxies, conforme coluna 1 na Tabela 3 ) levantados na literatura, uma vez que estes foram propostos por autores diferentes em contextos e momentos, também, distintos. Dessa forma, fatores utilizados em diferentes contextos podem se sobrepor ou compartilhar de uma mesma concepção intrínseca, o que pode gerar problemas de multicolinearidade numa análise conjunta da assimetria dos custos. Isto posto, as análises possibilitaram a extração de 6 dimensões (componentes, conforme Tabela 3) a partir da variância comum, ou seja, da explicação que os fatores compartilham na assimetria dos custos. As dimensões são formadas pela combinação de percentuais de cada um dos fatores explicativos e, dessa forma, possibilitaram uma análise mais assertiva e concisa para a explicação da assimetria dos custos, ao eliminar a multicolinearidade.

A principal diferença entre os achados exploratórios e confirmatórios está na associação entre os diferentes fatores explicativos, pois para algumas das dimensões da análise exploratória não há aporte teórico que as sustente. Essa diferença pode ter ocorrido devido às proxies numéricas e variáveis dummy utilizadas, uma vez que foram definidas com base na literatura e na disponibilidade dos dados.

\section{Conclusão}

A revisão de literatura desenvolvida por Banker, Byzalov, Fang e Liang (2018) evidenciou que ainda existe lacuna na literatura para discutir as descobertas empíricas dos estudos sobre comportamento dos custos como meio para a compreensão da tomada de decisão por parte dos gestores, os fatores institucionais e econômicos, ao atribuir uma visão mais ampla em vez do olhar isoladamente para os determinantes dos custos. Em decorrência, e ao se analisar a literatura sobre o tema, percebe-se que há indícios da existência de fatores que tendem a ser intercorrelacionados e que, em conjunto, expressam conceitos maiores que permeiam grupos de fatores denominados clusters. 
Assim, por meio da presente pesquisa, determinou-se a interação conjunta entre os fatores explicativos da assimetria dos custos, particularmente para o caso brasileiro, em dois grandes agrupamentos de fatores (cluster), que representam a dimensão do gestor, tratado como decisão deliberada dos gestores, e a dimensão da empresa, tratada como atraso nos ajustes de custos, que diz respeito à incapacidade dos custos serem ajustados rápido o suficiente para acompanhar as quedas nas receitas. Essas duas dimensões também são tratadas nos estudos de Yasukata e Kajiwara (2011) com as mesmas nomenclaturas, enquanto que Abdulhamied e Abulezz (2012) fazem referência a primeira dimensão como decorrente da incerteza na demanda (perspectiva dos gestores) e a segunda como estrutura de custos da organização (perspectiva da empresa).

Por meio da análise fatorial confirmatória identificou-se os pesos que os fatores explicativos possuem na formação dos clusters de forma a hierarquizá-los. Quanto ao aspecto relacionado ao decisor (decisão deliberada dos gestores), o fator que se apresentou mais significativo foi o fluxo de caixa, seguido pelo ambiente macroeconômico, otimismo, pessimismo dos gestores e, por fim, o problema de agência. Já, no que tange à estrutura operacional (atraso nos ajustes de custos), o fator que apresentou o maior peso foi custos de ajustamento, na sequência a intensidade de passivos, o tamanho das organizações, a capacidade ociosa, a magnitude das variações de receita e, por fim, a intensidade de ativos.

Ao analisar o impacto dos clusters na assimetria dos custos, conforme a metodologia proposta por Anderson, Banker e Janakiraman (2003), identificou-se que tanto a decisão deliberada dos gestores quanto o atraso nos ajustes de custos afetam positivamente, e em magnitudes semelhantes, a assimetria das despesas gerais (DG), ou seja, quanto maior a presença desses clusters nas empresas maior seu grau de assimetria. Contudo, para a análise dos custos dos produtos vendidos (CPV) e dos custos totais (CT) apenas o cluster de decisão deliberada dos gestores se mostrou significativo ao grau estabelecido de $90 \%$ de confiança. Tais resultados corroboram a visão contemporânea na gestão dos custos que reconhece que estes são causados pela decisão deliberada dos gestores e estão sujeitos tanto às características dos gestores de restrição, incentivos, expectativas e vieses psicológicos quanto ao contexto institucional e econômico em que se inserem (BANKER; BYZALOV; FANG ; LIANG, 2018).

A última análise desenvolvida na presente pesquisa identificou de forma exploratória 6 dimensões latentes, que resumem a estrutura de relações entre os fatores explicativos, ou seja, reduziu-se os 11 fatores explicativos (14 proxies) inseridos nas análises em 6 constructos que os representam. Contudo, nem todas as interações propostas na formação destas dimensões tem aporte teórico. Essa diferença entre a análise confirmatória e exploratória pode ser proveniente das proxies numéricas e das variáveis dummy utilizadas, uma vez que estas foram definidas com base na literatura e na disponibilidade de dados. Estes resultados evidenciam a necessidade de observar o comportamento dos custos com uma visão mais ampla e não isolada em relação aos fatores explicativos conforme já apontado na pesquisa de Banker, Byzalov, Fang e Liang (2018). Assim, os achados da pesquisa avançam em termos literários ao questionar a ocorrência de interações entre os fatores explicativos previamente identificados de forma isolada e que acabam por mensurar um único aspecto de diferentes maneiras.

Com base nos achados evidenciados é possível obter-se uma visão mais macro da forma como ocorre o comportamento assimétrico dos custos diante de suas causas levantadas. Logo, em vez de se observar apenas a influência do ambiente macroeconômico na assimetria, uma vez que este se associa a visão futura dos gestores, mais otimista ou pessimista em relação ao cenário, por exemplo, observa-se a totalidade - conjunta - dos fatores relacionados ao aspecto do decisor e da empresa. Deste modo, o presente estudo clarifica a existência dessas interações aos gestores e permite-lhes um maior controle sobre os custos e processos. 
Por fim, sugere-se uma investigação das interações citadas na literatura, por meio de um design de pesquisa que permita as análises sem restrição às informações financeiras disponíveis, a exemplo de uma análise qualitativa, por meio de um estudo de caso ou entrevistas para dar maior sustentação às comparações das dimensões do decisor e da empresa.

\section{Referências}

ABDULHAMIED, A. A.; ABULEZZ, M. E. An empirical investigation of the effect of cost structure and demand uncertainty on $S A \& G$ cost stickiness. Islamic Business Researches Center, p. 1-27, 2012.

ANDERSON, M. C.; BANKER, R. D.; JANAKIRAMAN, S. N. Are selling, general, and administrative costs "sticky"? Journal of Accounting Research, v. 41, n. 1, p. 47-63, 2003. https://doi.org/10.1111/1475-679X.00095

AZEEZ, K. A.; DONGPING, H.; MAHMOOD, M. A. Capacity expansion decisions into asymmetric cost behaviour: reviews and search for new determinants. International Journal of Services Operations and Informatics, v. 9, n. 2, p. 139-159, 2018. https://doi.org/10.1504/IJSOI.2018.092585

AZEEZ, K. A; DONGPING, H.; DONATA, S. The Moderating Effects of Capacity Utilization on the Relationship Between Capacity Changes and Asymmetric Labor Costs Behavior. In: International Conference on Management Science and Engineering Management. Springer, Cham, 2017. p. 260-273. https://doi.org/10.1007/978-3-319-592800_21

BALAKRISHNAN, R.; GRUCA, T. S. Cost stickiness and core competency: A note. Contemporary Accounting Research, v. 25, n. 4, p. 993-1006, 2008. https://doi.org/10.1506/car.25.4.2

BALAKRISHNAN, R.; LABRO, E.; SODERSTROM, N. S. Cost structure and sticky costs. Journal of Management Accounting Research, v. 26, n. 2, p. 91-116, 2014. https://doi.org/10.2308/jmar-50831

BALAKRISHNAN, R.; PETERSEN, M. J.; SODERSTROM, N. S. Does capacity utilization affect the "stickiness" of cost? Journal of Accounting, Auditing \& Finance, v. 19, n. 3, p. 283-299, 2004. https://doi.org/10.1177/0148558X0401900303

BANKER, R. D.; BYZALOV, D. Asymmetric cost behavior. Journal of Management Accounting Research, v. 26, n. 2, p. 43-79, 2014. https://doi.org/10.2308/jmar-50846

BANKER, R. D.; CHEN, L. Predicting earnings using a model based on cost variability and cost stickiness. The Accounting Review, v. 81, n. 2, p. 285-307, 2006.

https://doi.org/10.2308/accr.2006.81.2.285

BANKER, R. D.; BYZALOV, D.; CIFTCI, M.; MASHRUWALA, R. The moderating effect of prior sales changes on asymmetric cost behavior. Journal of Management Accounting Research, v. 26, n. 2, p. 221-242, 2014. https://doi.org/10.2308/jmar-50726 
BANKER, R. D.; BYZALOV; D., FANG, S.; LIANG, Y. Cost management research. Journal of Management Accounting Research, v. 30, n. 3, p. 187-209, 2018. https://doi.org/10.2308/jmar-51965

BANKER, R. D.; CIFTCI, M.; MASHRUWALA, R. Managerial optimism, prior period sales changes, and sticky cost behavior. Working Paper, Temple University, 2008. Disponível em: https://papers.ssrn.com/sol3/papers.cfm?abstract_id=1599284. Acesso em: 10 nov. 2015.

BANKER, R.; FANG, S.; MEHTA, M. Real cost management and anomalous financial performance ratios during economic downturns. Working paper, Temple University, 2013.

BLUE, G.; MOAZEZ, E.; KHANHOSSINI, D.; NIKOONESBATI, M. The Relationship between Perspective Managers and' Sticky Costs' in the Tehran Stock Exchange. Social Science Research Network, 2013. Disponível em:

https://papers.ssrn.com/sol3/papers.cfm?abstract_id=2216631. Acesso em: 10 nov. 2015.

CALLEJA, K.; STELIAROS, M.; THOMAS, D. C. A note on cost stickiness: Some international comparisons. Management Accounting Research, v. 17, n. 2, p. 127-140, 2006. https://doi.org/10.1016/j.mar.2006.02.001

CHEN, Jason V.; KAMA, Itay; LEHAVY, Reuven. A contextual analysis of the impact of managerial expectations on asymmetric cost behavior. Review of Accounting Studies, v. 24, n. 2, p. 665-693, 2019. https://doi.org/10.1007/s11142-019-09491-2

CHEN, C. X.; LU, H.; SOUGIANNIS, T. The agency problem, corporate governance, and the asymmetrical behavior of selling, general, and administrative costs. Contemporary

Accounting Research, v. 29, n. 1, p. 252-282, 2012. https://doi.org/10.1111/j.19113846.2011.01094.x

DALLA VIA, N.; PEREGO, P. Sticky cost behaviour: evidence from small and medium sized companies. Accounting \& Finance, v. 54, n. 3, p. 753-778, 2014.

https://doi.org/10.1111/acfi.12020

FÁVERO, L. P.; Belfiore, P., Da Silva, F. L., Chan, B. L. Análise de dados: modelagem multivariada para tomada de decisões. Rio de Janeiro: Elsevier, 2009.

HAIR JR, J. F; HULT, G. T. M.; RINGLE, C.; SARSTEDT, M. A primer on partial least squares structural equation modeling (PLS-SEM). California: Sage Publications, 2016.

KAMA, I.; WEISS, D. Do earnings targets and managerial incentives affect sticky costs? Journal of Accounting Research, v. 51, n. 1, p. 201-224, 2013. https://doi.org/10.1111/j.1475-679X.2012.00471.x

KRISHNAN, R. Management accountant - What ails thee? Journal of Management Accounting Research, v. 27, n. 1, p. 177-191, 2015. https://doi.org/10.2308/jmar-10461

LIU, Xiaotao; LIU, Xiaoxia; REID, Colin D. Stakeholder orientations and cost management. Contemporary Accounting Research, v. 36, n. 1, p. 486-512, 2019. https://doi.org/10.1111/1911-3846.12389 
LOWRY, P. B.; GASKIN, J. Partial least squares (PLS) structural equation modeling (SEM) for building and testing behavioral causal theory: When to choose it and how to use it. IEEE transactions on professional communication, v. 57, n. 2, p. 123-146, 2014. https://10.1109/TPC.2014.2312452

MALIK, M. A review and synthesis of 'cost stickiness' literature. Social Science Research Network, 2012. Disponível em: http://ssrn.com/abstract=2276760. Acesso em: 10 nov. 2015.

MEDEIROS, O. R. de; COSTA, P. de S.; SILVA, C. A. T. Testes empíricos sobre o comportamento assimétrico dos custos nas empresas brasileiras. Revista Contabilidade \& Finanças, v. 16, n. 38, p. 47-56, 2005. https://doi.org/10.1590/S1519-70772005000200005

NAMITHA, C.; SHIJIN, S., 2016. Managerial discretion and agency cost in Indian market. Advances in accounting, 35, 159-169. https://doi.org/10.1016/j.adiac.2016.06.002

NASSIRZADEH, F.; SAEI, M. J.; SALEHI, M. A Study of the stickiness of cost of goods sold and operating costs to changes in sales level in Iran. Studies in Business and Economics, v. 8, n. 2, p. 79-89, 2013.

PAMPLONA, E.; FIIRST, C., SILVA, T. B. J.; ZONATTO, V. C. S. Comportamento dos custos das maiores empresas do Brasil, Chile e México entre 2002 a 2013 e análise dos sticky costs. In: Congresso Anpcont, 9, 2015, Curitiba. Anais... Anpcont, 2015.

RICHARTZ, F.; BORGERT, A. O comportamento dos custos das empresas brasileiras listadas na BM\&FBovespa entre 1994 e 2011 com ênfase nos sticky costs. Contaduría y administración, v. 59, n. 4, p. 39-70, 2014. https://doi.org/10.1016/S0186-1042(14)70154-8

RICHARTZ, F.; BORGERT, A. Modelo explicativo para o comportamento assimétrico dos custos das empresas brasileiras. In: Congresso Anpcont, 9, 2015, Curitiba. Anais... Anpcont, 2015.

RICHARTZ, F.; BORGERT, A.; LUNKES, R. J. Comportamento Assimétrico dos Custos nas empresas Brasileiras listadas na BM\&FBovespa. Advances in Scientific and Applied Accounting, v. 7, n. 3, p. 339-361, 2014. https://doi.org/10.14392/ASAA.2014070302 SUBRAMANIAM, C.; WEIDENMIER, M. Additional evidence on the behavior of sticky costs. Advances in Management Accounting, v. 26, p. 275-305, 2016.

YASUKATA, Kenji. Are 'Sticky Costs' the Result of Deliberate Decision of Managers? Social Science Research Network, 2011. http://dx.doi.org/10.2139/ssrn.1444746 